\title{
La visibilidad de los resultados de la investigación de los programas de posgrado de la UNAH
}

CIENCIAYTECNOLOGÍA(CT) Entrevista a:

Ramón Romero (RR): Realizó sus estudios de Licenciatura en Ciencias Jurídicas y Sociales, egresado de la Maestría en Filosofía, con estudios de Doctorado en Filosofía. Actualmente es el Director de Vinculación Universidad-Sociedad de la Universidad Nacional Autónoma de Honduras.

Ramón Salgado (RS): Realizó sus estudios de Licenciatura en Sociología, egresado de la Maestría en Sociología. Actualmente es el Director de la Dirección de Educación Superior y coordinador de la asignatura de Sociología en la Facultad de Ciencias Sociales de la Universidad Nacional Autónoma de Honduras.

CT/ Desde su punto de vista ¿en qué estado se encuentra la investigación en los posgrados de la UNAH? ¿se puede hacer alguna diferencia por área de conocimiento?

RR/ Sí, primero una consideración general en el sentido de que, durante mucho tiempo, los procesos de investigación en la universidad han sido muy limitados. Tenemos un grave déficit de investigación, ese déficit se ha empezado a solventar con el programa de la Cuarta Reforma Universitaria. A través de la Dirección de Investigación Científica se realiza una labor muy intensa para lograr que la investigación sea dentro de la universidad, una función académica cada vez más importante; en los posgrados se ha padecido bastante de ese déficit general. Creo que sí se pueden hacer unas diferencias en cuanto a áreas del conocimiento; de hecho, hay reconocimiento en el área de la salud, particularmente en la Facultad de Ciencias Médicas hay un considerable nivel de investigación, también en la carrera y la Escuela de Microbiología y otras unidades académicas que manejan procesos de investigación con cierta intensidad, pero que no llegan a los niveles en que se encuentran Medicina y Microbiología; aquí se pueden identificar algunos posgrados en las áreas de las ciencias sociales, entre los que se puede citar el posgrado de PLATS, seguramente a través de las tesis del doctorado en gestión en desarrollo y quizás a nivel de algunas tesis del posgrado centroamericano en Economía y Desarrollo. 
RS/ Existen dos tipos de posgrados en América Latina, los profesionalizantes y los de investigación. En caso de la UNAH y buena parte de las Instituciones de Educación Superior (IES) del país, predominan los posgrados profesionalizantes y son muy pocos los orientados a la investigación científica, por lo tanto, no se puede esperar que con este tipo de posgrados se tenga una cantidad suficiente de investigaciones y publicaciones a nivel internacional. En el caso de las ciencias de la salud, se observa que los posgrados hacen mayor énfasis en trabajos de investigación y por lo tanto la cantidad de investigaciones y su publicación a nivel internacional es más evidente.

\section{CT/ ¿Considera usted que tienen calidad los procesos investigativos desarrollados en los posgrados a nivel de trabajos específicos en las distintas asignaturas y tesis?}

RR/ Esta respuesta no se puede asumir de manera generalizada; hay distintos niveles diferenciados tenemos tesis que, dependiendo de los asesores y de la formación que los estudiantes hayan tenido en los procesos de investigación, pueden tener más calidad que otras y éste no es un problema de unas escuelas 0 posgrados, es un problema de los asesores, la educación de los estudiantes y las ternas examinadoras que juegan un papel importante como filtro para determinar la calidad; los asesores de tesis son muy permisivos y si las ternas examinadoras no cumplen a cabalidad su papel, entonces considerablemente habrá una merma en la calidad de las tesis.

RS/ Los trabajos de investigación de las asignaturas de posgrados deben considerarse como experiencias básicas para familiarizar a los estudiantes tanto en la investigación cualitativa como en la investigación cuantitativa. Lo deseable es que de estas investigaciones de aula surjan problemas de investigación más significativos que puedan ser desarrollados en sus trabajos de tesis con pertinencia y trascendencia nacional e internacional.

CT/ Entre los programas de los posgrados, ¿existen a su criterio, investigaciones destacables? ¿podría mencionarlas y decir en qué criterio se basa para otorgarles ese valor?

RR/ Sí, desde luego que hay investigaciones destacables, particularmente las investigaciones en el campo de la neurociencia y en el campo de la psiquiatría que se han conducido en la Escuela de Medicina; merecen destacarse y el criterio fundamental para otorgarles ese valor son los premios de investigación científica 


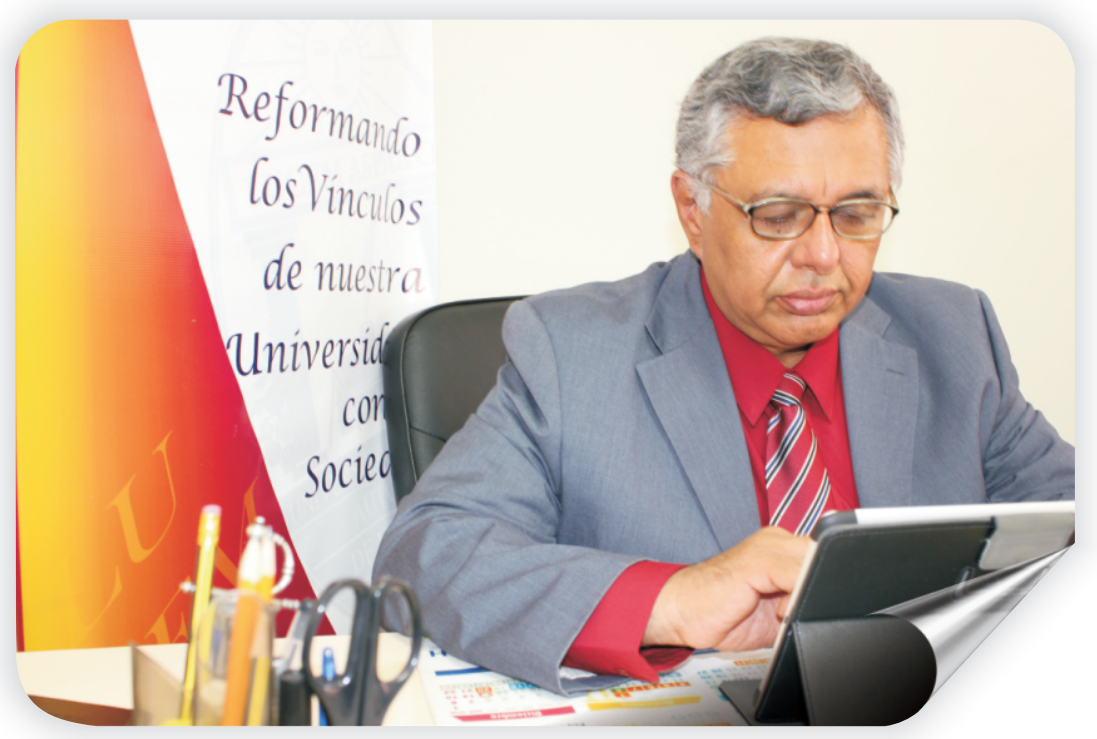

Ramón Romero

que ha otorgado la Universidad a la Facultad de Medicina por sus trabajos en neurociencia a través del Dr. Marco Tulio Medina y de psiquiatría a través del Dr. Américo Reyes Ticas. En el área de microbiología hay trabajos importantes que han sido premiados, por ejemplo, el Premio de Investigación conferido a la Dra. Ivette Lorenzana de Rivera y el último premio que se le otorgó en el área de las Ciencias Sociales a la Dra. Elsa Lily Caballero. Sobre esta base podemos entonces, hablar de investigaciones destacables.

RS/ Los programas de posgrados de Ciencias de la Salud y de la carrera de Microbiología destacan con investigaciones relevantes y que han sido premiadas por el Consejo Superior Universitario, CSUCA. Además en el ranking iberoamericano de Scimago, la posición de la UNAH se logra en su mayoría por investigaciones desarrolladas en estos campos del conocimiento.

\section{$\mathrm{CT} /$ ¿Cuáles son las limitaciones para que profesores y estudiantes de los posgrados hagan investigación y publiquen?}

RR/ En mi criterio, la limitación es de carácter cultural: hay poca propensión a la investigación; a ello se pueden agregar otras limitaciones que son menores como: la formación en la investigación, el tiempo que se dedica a la investigación, y las limitaciones metodológicas que los estudiantes puedan tener; creo que todas estas 
cosas son subsanables sin mayor problema y el tema central, repito, es el tema de la cultura de investigación en la academia universitaria que no ha logrado, hasta ahora, un desarrollo importante.

RS/ La concepción en la que están estructurados los posgrados. Se deben definir los posgrados de investigación y los posgrados profesionalizantes. En ambos casos se debe hacer investigación pero la naturaleza de los mismos es diferente. La UNAH debe apostar más por los posgrados de investigación, los que deben tener un currículum elaborado para tal efecto, y dar todo el apoyo al cuerpo de profesores y estudiantes para que investiguen y publiquen nacional e internacionalmente. Los graduados de este tipo de posgrados de manera natural deberían pasar a los estudios de doctorado, mientras los profesionales, previo a ingresar a un doctorado debieran hacer más cursos y obtener más experiencia en investigación.

\section{$\mathrm{CT} /$ Según su criterio, ¿Qué rol deben desempeñar los profesores y asesores en el proceso de elaboración de la investigación?}

RR/ Creo que tienen que jugar varios papeles: primero, de guía metodológica; segundo, de animador de la investigación y animador del estudiante; tercero, de contralor, para que el plan de investigación se vaya ejecutando con todos los requerimientos y en los tiempos que están siendo planteados; luego, tiene un rol de facilitador en el sentido de procurar que el estudiante pueda superar los problemas formales, inclusive los burocráticos, que pueden encontrarse en el trabajo de confección de su tesis y, por otra parte, el profesor tiene que ser un orientador en los contenidos de las tesis, no solamente en la metodología y aquí la orientación principal es para que en las tesis de posgrado se hagan contribuciones a la teoría, eso es fundamental si se está desarrollando un tema cualquiera, este tema va más allá de un diagnóstico, una evaluación o un estudio de factibilidad. Eso es muy limitado para una tesis de posgrado pues, por muy modesta que sea, debe hacer una contribución original en el campo en la cual se está trabajando.

RS/ Cada uno de los profesores y asesores de tesis deben tener proyectos de investigación, y los alumnos deben conocerlos y vincularse a estos proyectos para elaborar sus trabajos de investigación o tesis. El profesor debe disponer de fondos para financiar sus temas de investigación a mediano y largo plazo. 


\section{CT/ ¿Qué recomendaciones haría a los gestores de programas de posgrado para lograr mejores resultados en la investigación?}

RR/ La recomendación fundamental es que se enfatice en la formación para la investigación; en los programas de posgrado debe haber un componente epistemológico, un componente metodológico y un componente técnico de investigación; esto posibilita que los estudiantes tengan las herramientas que necesitan para ejecutar los programas o los procesos de investigación. Por otra parte, que en los distintos seminarios y actividades de los posgrados haya un nivel de exigencia en la formulación de trabajos que requieran investigación, de tal manera que los seminarios u otros procesos académicos debieran concluir con la presentación de investigaciones cortas, pero que ejerciten al estudiante en la metodología y las técnicas de investigación y que, además, vayan constituyendo avances para la formulación de sus tesis de posgrado; y una tercera recomendación es que los gestores de programas vinculen la política de investigación de sus programas con la política de investigación de toda la UNAH y con las necesidades en materia de investigación que existen en el país.

RS/ Cambiar de paradigma y hacer una revisión de lo que están haciendo las universidades exitosas en investigación. Lo que hace la Universidad de Sao Pablo, Brasil, es un buen punto de referencia.

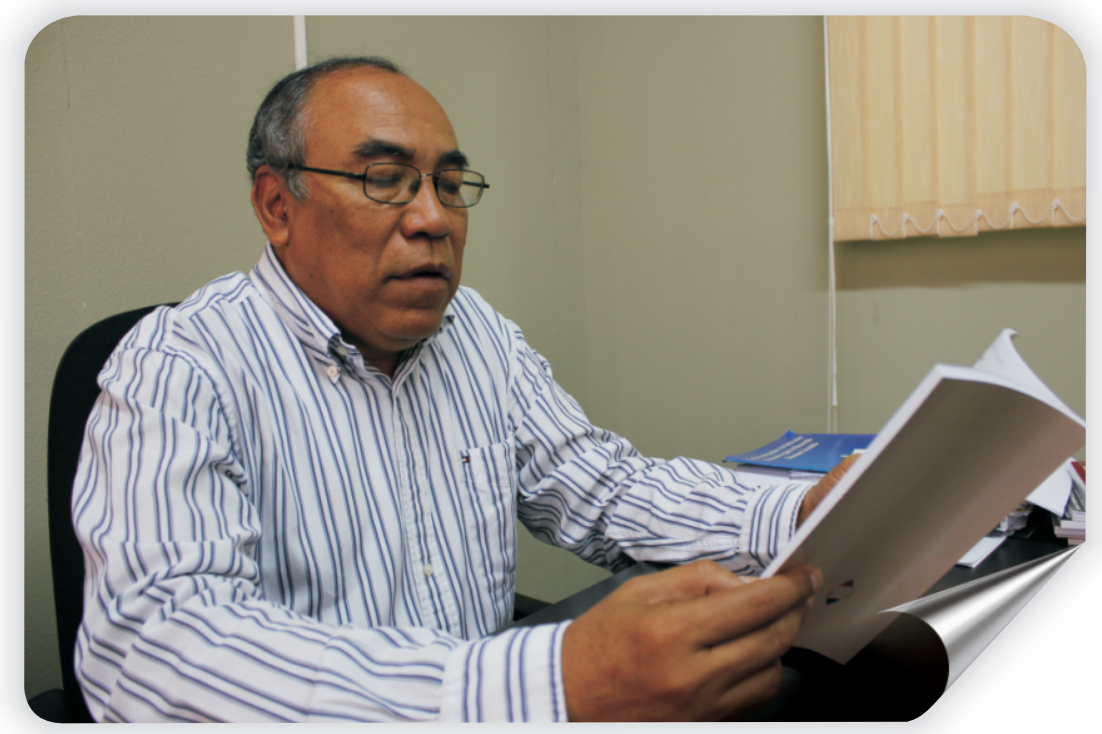

Ramón Salgado 


\section{$\mathrm{CT} /$ ¿Qué recomendaciones nos sugiere para visibilizar los resultados de las investigaciones de posgrado?}

RR/ De nuevo varias cosas: primero, que las investigaciones valiosas tienen que publicarse de manera que lleguen a quienes estarían demandando esos conocimientos; por ejemplo, las investigaciones o tesis que se va a publicar sobre vivienda, deben ser del conocimiento de académicos, funcionarios públicos, ONG's y sectores productivos que trabajan en el tema, de manera que el conocimiento formulado en la tesis pueda volverse útil. Otra recomendación para visualizar los resultados es hacer presentaciones, debates y mesas redondas sobre temas de investigación que permitan desarrollar, por una parte, difusión, y por otra, complementariedad entre distintas tareas de investigación.

RS/ Crear un cuerpo de lectores o jueces de los trabajos de investigación y que cuando se publiquen, cuenten con una aprobación académica y darle la mayor difusión posible.

\section{CT/ ¿Cómo deben ser definidos los temas de investigación de las tesis de los posgrados? ¿deben orientarse a temas prioritarios de investigación para concentrar esfuerzos dispersos, o no?}

RR/ Pienso que deben concentrarse en los temas prioritarios. La universidad ha establecido su apuesta dentro de los 13 temas prioritarios de investigación, y ellos deben ser abordados por los posgrados, desde sus particularidades; luego, debe revisarse de manera permanente la pertinencia entre las prioridades definidas por la universidad y las prioridades del país en materia de investigación y en razón de los resultados de vincular unas con otras y ver la pertinencia de unas y otras. A partir de allí pueden identificarse los temas de investigación, de manera que estos estén siempre dentro de las prioridades para que la investigación pueda ser útil; debemos evitar los esfuerzos dispersos en los cuales se investiga sobre casi cualquier cosa, pensando en qué es lo que está más a mano o lo que pueda resultar más fácil o más agradable, debe concentrarse el proceso en el marco de las prioridades de la investigación.

RS/ Se deben utilizar dos criterios: Las prioridades establecidas por la UNAH en su plan general de investigación, acorde a las necesidades del país. Y el segundo, insertarse en un proyecto de investigación que ya esté en marcha por algún profesor universitario y que lo pueda acompañar en el desarrollo de la tesis. 


\section{$\mathrm{CT} /$ ¿Considera que existen debilidades metodológicas y técnicas para hacer más y mejor investigación en los posgrados? ¿Qué propone para superarlas?}

RR/ Posiblemente haya debilidades metodológicas, pero más que juzgar si hay o no debilidades metodológicas, lo importante es enfatizar que tiene que haber un proceso permanente de mejora en los métodos y técnicas de investigación en los posgrados. Sea que tengan un proceso débil de investigación o sea que estén fortalecidos los temas metodológicos, epistemológicos y técnicos, deben estarse tratando siempre en los posgrados, de manera que eso garantice que se superen debilidades o se mejore la calidad de la investigación de manera constante; ello se logra a través de seminarios o cursos distintos, mecanismo que en los posgrados se ejecuta para que este tema sea atendido con la importancia que tiene. Jamás debe olvidarse que un elemento esencial, un componente básico de los posgrados son las investigaciones; un posgrado en el que no se realicen investigaciones se degrada cada vez más hasta que deja de ser un posgrado de calidad.

RS/ Al momento del desarrollo de las investigaciones se tienen que identificar las necesidades de capacitación de los estudiantes y desarrollar talleres especiales sobre metodologías, redacción de textos científicos o los temas sobre los cuales se requiere formación.

\section{CT/ ¿A qué se debe la ineficiencia terminal de los posgrados? ¿Cuáles son las razones por la que un estudiante no termina el plan de estudio de un posgrado con la tesis lista? ¿Qué lo impide?}

RR/ Yo he tenido conocimiento y experiencia con estudiantes de posgrado en nuestra universidad en el área de ciencias sociales. La explicación del porqué las tesis nunca llegan a terminarse, primero, porque mientras se estaba cursando el posgrado no se avanzó lo suficiente en la formulación de ésta; si logramos desarrollar las actividades académicas en los posgrados y un gran avance en la tesis, podemos lograr que lo que quede por hacer sea relativamente poco y, además, que quede bastante claro lo que está pendiente de hacer. Segundo, los directores de tesis descuidan el proceso de preparación de la tesis de los estudiantes y decíamos que un director de tesis debe ser entre otras cosas un orientador y un motivador, además de un supervisor; quizá una tercera razón es que, a medida que se van desvinculando de los posgrados, los estudiantes o los ex estudiantes van asumiendo otro tipo de responsabilidades de carácter laboral o de distinto tipo y ello puede determinar que las condiciones para trabajar en la tesis vayan siendo cada vez más precarias y difíciles, razón por la cual los posgrados 
deben insistir en darle seguimiento a los estudiantes al egresar de los posgrados y exigirles un periodo de tiempo para la presentación de la misma.

RS/ Existen varias razones. La primera es que la estructura de los currículos no considera la formación de investigación como un eje transversal, que va desde el inicio hasta la parte final; en segundo lugar, no se cuenta con un cuerpo de profesores investigadores que oriente el trabajo de los estudiantes y tercero, se eligen temas sobre los cuáles no se tiene suficiente información y recursos para desarrollarlos en el tiempo que establecen los planes de estudio y, finalmente, se debe desarrollar la disciplina en los estudiantes para cumplir con el proceso. 